\title{
Extension of energy density analysis to periodic-boundary-condition calculations with plane-wave basis functions
}

\author{
Yutaka Imamura, ${ }^{1}$ Asuka Takahashi, ${ }^{1}$ Takeshi Okada, ${ }^{1}$ Takahisa Ohno, ${ }^{2}$ and Hiromi Nakai ${ }^{1,3, *}$ \\ ${ }^{1}$ Department of Chemistry and Biochemistry, School of Advanced Science and Engineering, Waseda University, 3-4-1 Okubo, \\ Shinjuku-ku, Tokyo 169-8555, Japan \\ ${ }^{2}$ National Institute for Materials Science, 1-2-1 Sengen, Tsukuba, Ibaraki 305-0045, Japan \\ ${ }^{3}$ Research Institute for Science and Engineering, Waseda University, 3-4-1 Okubo, Shinjuku-ku, Tokyo 169-8555, Japan
}

(Received 3 June 2009; revised manuscript received 20 November 2009; published 26 March 2010)

\begin{abstract}
Energy density analysis (EDA), which partitions the total energy of a molecular system into atomic contributions, has been extended to periodic-boundary-condition calculations carried out by density-functional theory using plane-wave (PW) basis functions and pseudopotentials. The present analysis method, called PW-EDA, allows us to extract local-energy information by estimating atomic energy contributions. Numerical applications to an isolated molecule $\mathrm{H}_{2} \mathrm{O}$ and the adsorption of $\mathrm{C}_{2} \mathrm{H}_{2}$ on the $\mathrm{Si}(001)-(2 \times 1)$ surface confirm the reliability and usefulness of PW-EDA.
\end{abstract}

DOI: 10.1103/PhysRevB.81.115136

PACS number(s): 73.20.Hb, 73.20.At

\section{INTRODUCTION}

Electronic structures on crystals and surfaces, i.e., periodic systems, are treated differently in solid-state physics and quantum chemistry. In solid-state physics, densityfunctional theory (DFT) with plane-wave (PW) basis functions has been used since the fast Fourier transform (FFT) reduces the computational cost significantly, and PW basis functions are suitable to describe the periodicity of solids. ${ }^{1}$ The PW-based DFT calculations are combined with pseudopotentials, which replace core electrons. This approach is based on a periodic model under the periodic boundary condition (PBC).

In quantum chemistry, wave-function theory such as Hartree-Fock and second-order perturbation theory has been used because of the accurate description. The linear combination of atomic orbitals (AOs) approximation, which expands molecular orbitals in terms of AOs, has been adopted. Gaussian-type orbitals (GTOs) are widely used as an AO due to feasible computation of analytical two-electron integrals. This approach is based on a finite cluster model as a model of crystals and surfaces.

After the improvement in exchange-correlation functionals in DFT, i.e., development of the generalized gradient approximation (GGA) (Refs. 2-4) and hybrid functionals, ${ }^{5}$ DFT has become a mainstream method even in quantum chemistry. Also, the PBC models with GTOs have been developed. ${ }^{6-9}$ Thus, the difference in the computational methods between solid-state physics and quantum chemistry seems to be basis functions: PW and GTO basis functions.

The PBC calculations are also applicable to investigate local phenomena in solids such as defects and chemical reactions. Local information needs to be extracted from a whole system in order to analyze local phenomena. The extraction strongly depends on the choice of basis functions. For GTO-based PBC calculations, we can take advantage of information of AOs since the centers of GTOs are set to the centers of nuclei and, therefore, the estimation of atomic contribution from molecular orbitals is easily performed. Thus, atomic populations ${ }^{10}$ and energy partitioning in terms of atoms ${ }^{11}$ and bonds, ${ }^{12}$ which are based on information of AOs, are common. However, the partitioning depends on GTO basis functions owing to the use of information of AOs. Thus, space partitioning functions have been also examined. ${ }^{13,14}$

On the other hand, analysis techniques of PW-based PBC calculations such as the projected density of states are limited in comparison with the GTO-based calculations owing to the lack of the centers of PW basis functions. Moreover, most of the previous analysis methodologies focus on the electron density. The analysis methodology, which is easy to implement and clear in interpretation, should be developed in order to obtain new local information from PW-based PBC calculations. The energy partitioning offers a new analysis methodology that is useful to analyze physical and chemical phenomena in terms of the atomic-energy changes. Thus, we propose PW-based energy density analysis (PW-EDA), which is an alternative energy partitioning using the spacepartitioning function for PW-based calculations.

\section{THEORY}

The total DFT energy $E$ with PW basis functions and pseudopotentials in the PHASE program ${ }^{15}$ is defined as

$$
E_{\mathrm{TOT}}=E_{\mathrm{KIN}}+E_{\mathrm{L}}+E_{\mathrm{NL}}+E_{\mathrm{CLM}}+E_{\mathrm{XC}}+E_{\mathrm{ION}-\mathrm{ION}} .
$$

$E_{\mathrm{KIN}}, E_{\mathrm{L}}$, and $E_{\mathrm{NL}}$ represent the kinetic energy, local, and nonlocal ion core-electron interactions, respectively. $E_{\mathrm{XC}}$, $E_{\mathrm{CLM}}$, and $E_{\mathrm{ION}-\mathrm{ION}}$ are the DFT exchange-correlation functional, the Coulomb, and ion-ion core interactions.

Assume that the $i$ th orbital is expressed in terms of PW basis functions

$$
\left|\phi_{\mathbf{k}}^{i}\right\rangle=\frac{1}{\sqrt{\Omega}} \sum_{\mathbf{G}} c_{\mathbf{k}+\mathbf{G}}^{i} \exp [i(\mathbf{k}+\mathbf{G}) \cdot \mathbf{r}],
$$

where $c_{\mathbf{k}+\mathbf{G}}^{i}, \Omega, \mathbf{r}, \mathbf{k}$, and $\mathbf{G}$ represent an expansion coefficient, the volume of a unit cell, the electron coordinate, a $k$ point, and a reciprocal lattice vector, respectively. The energy expressions of the above terms using Eq. (2) are given as 


$$
\begin{aligned}
& E_{\mathrm{KIN}}=\frac{1}{2} \sum_{i \mathbf{k}} \sum_{\mathbf{G}}\left|c_{\mathbf{k}+\mathbf{G}}^{i}\right|^{2}|\mathbf{k}+\mathbf{G}|^{2}, \\
& E_{\mathrm{L}}=\Omega \sum_{\mathbf{G} \neq 0} \sum_{\mathrm{A}}^{\text {atom }} \rho(\mathbf{G}) V_{\mathrm{A}}^{\mathrm{loc}}(\mathbf{G}) \exp \left(-i \mathbf{G} \cdot \mathbf{R}_{\mathrm{A}}\right), \\
& E_{\mathrm{NL}}=\sum_{\mathrm{A}}^{\text {atom }} \sum_{l m} \sum_{\tau \tau^{\prime}} D_{\tau \tau^{\prime} l}^{\mathrm{A} \text { ion }} \sum_{i \mathbf{k}} f_{\tau l m \mathbf{k}}^{\mathrm{A}, i^{*}} f_{\tau \prime l m \mathbf{k}}^{\mathrm{A}, i} \\
& E_{\mathrm{CLM}}=2 \pi \Omega \sum_{\mathbf{G} \neq 0} \frac{\rho(-\mathbf{G}) \rho(\mathbf{G})}{|\mathbf{G}|^{2}}, \\
& E_{\mathrm{XC}}=\sum_{\mathrm{g}} \omega_{\mathrm{g}} \varepsilon_{\mathrm{XC}}\left[\rho\left(\mathbf{r}_{\mathrm{g}}\right)\right] \rho\left(\mathbf{r}_{\mathrm{g}}\right), \\
& E_{\mathrm{ION}-\mathrm{ION}}=\sum_{\mathrm{A}} \sum_{\mathrm{B}}^{\text {atom atom }} Z_{\mathrm{A}} Z_{\mathrm{B}}\left[\frac { 4 \pi } { \Omega } \left(\sum_{\mathbf{G} \neq 0} \frac{e^{-(\alpha|\mathbf{G}| / 2)^{2}}}{|\mathbf{G}|^{2}} e^{i \mathbf{G} \cdot\left(\mathbf{d}_{\mathrm{A}}-\mathbf{d}_{\mathrm{B}}\right)}\right.\right. \\
& \left.\left.-\frac{\alpha^{2}}{4}\right)+\sum_{\mathbf{L}} \frac{\operatorname{erfc}\left(\left|\mathbf{d}_{\mathrm{A}}-\mathbf{d}_{\mathrm{B}}-\mathbf{L}\right| / \alpha\right)}{\left|\mathbf{d}_{\mathrm{A}}-\mathbf{d}_{\mathrm{B}}-\mathbf{L}\right|}-\frac{2}{\alpha \sqrt{\pi}} \delta_{\mathrm{A}, \mathrm{B}}\right],
\end{aligned}
$$

where $Z_{\mathrm{A}}, \mathbf{R}_{\mathrm{A}}$, and $\mathbf{g}$ are the nuclear charge, the nucleus coordinate, and a grid, respectively. $\rho(\mathbf{G})$ and $V_{\mathrm{A}}^{\text {loc }}(\mathbf{G})$ are the Fourier components of the density $\rho(\mathbf{r})$ and local ion coreelectron interaction $V_{\mathrm{A}}^{\mathrm{loc}}(\mathbf{r})$. The details of $E_{\mathrm{NL}}$ and $E_{\mathrm{ION}-\mathrm{ION}}$ are given in Ref. 21.

The present study proposes a term-by-term partitioning scheme of the total energy expressed in Eq. (1) into atomic contributions

$$
\begin{aligned}
E_{\mathrm{TOT}}= & \sum_{\mathrm{A}} E^{\mathrm{A}}=\sum_{\mathrm{A}}\left[\left(E_{\mathrm{NL}}^{\mathrm{A}}+E_{\mathrm{ION}-\mathrm{ION}}^{\mathrm{A}}\right)+E_{\mathrm{XC}}^{\mathrm{A}}+\left(E_{\mathrm{KIN}}^{\mathrm{A}}+E_{\mathrm{L}}^{\mathrm{A}}\right.\right. \\
& \left.\left.+E_{\mathrm{CLM}}^{\mathrm{A}}\right)\right] .
\end{aligned}
$$

Here, each term is categorized into three groups, which are divided by parentheses in Eq. (9).

The first group consists of two terms, i.e., the nonlocal ion core-electron interaction $E_{\mathrm{NL}}$ and ion-ion core interaction $E_{\text {ION-ION, which are essentially based on their atomic summa- }}$ tion. Namely, they are evaluated by summing over atoms. Therefore, atomic contributions are estimated by taking advantage of the information of their atomic summations. The atomic contribution of the nonlocal ion core-electron interaction $E_{\mathrm{NL}}$ and ion-ion core interaction $E_{\mathrm{ION}-\mathrm{ION}}$ are defined as follows:

$$
\begin{gathered}
E_{\mathrm{NL}}^{\mathrm{A}}=\sum_{l m} \sum_{\tau \tau^{\prime}} D_{\tau \tau^{\prime} l}^{\mathrm{A} \text { ion }} \sum_{i \mathbf{k}} f_{\tau l m \mathbf{k}}^{\mathrm{A}, i^{*}} f_{\tau \prime l m \mathbf{k}}^{\mathrm{A}, i}, \\
E_{\mathrm{ION}-\mathrm{ION}}^{\mathrm{A}}=\sum_{\mathrm{B}} Z_{\mathrm{A}} Z_{\mathrm{B}}\left[\frac{4 \pi}{\Omega}\left(\sum_{\mathbf{G} \neq 0} \frac{e^{-(\alpha|\mathbf{G}| / 2)^{2}}}{|\mathbf{G}|^{2}} e^{i\left(\mathbf{G} \cdot\left(\mathbf{d}_{\mathrm{A}}-\mathbf{d}_{\mathrm{B}}\right)\right.}-\frac{\alpha^{2}}{4}\right)\right. \\
\left.+\sum_{\mathbf{L}} \frac{\operatorname{erfc}\left(\left|\mathbf{d}_{\mathrm{A}}-\mathbf{d}_{\mathrm{B}}-\mathbf{L}\right| / \alpha\right)}{\left|\mathbf{d}_{\mathrm{A}}-\mathbf{d}_{\mathrm{B}}-\mathbf{L}\right|}-\frac{2}{\alpha \sqrt{\pi}} \delta_{\mathrm{A}, \mathrm{B}}\right]
\end{gathered}
$$

The second group is only one term: the exchangecorrelation functional $E_{\mathrm{XC}}$, which is evaluated using grid points in the real-space representation. The normal PW-PBC calculations do not require atomic information since they use grid points determined homogeneously. On the other hand, GTO-PBC calculations adopt grid points determined and centered at each nucleus, which may lead to double counting problem. Thus, the Becke's partitioning function $p_{\mathrm{A}},{ }^{13}$ which satisfies the following fundamental conditions:

$$
p_{\mathrm{A}}(\mathbf{r}) \geq 0
$$

and

$$
\sum_{\mathrm{A}} p_{\mathrm{A}}(\mathbf{r}) \equiv 1
$$

has been introduced. Here, we use the Becke's partitioning function $p_{\mathrm{A}}$ in order to estimate atomic contributions

$$
E_{\mathrm{XC}}^{\mathrm{A}}=\sum_{\mathrm{g}} \omega_{\mathrm{g}} p_{\mathrm{A}}\left(\mathbf{r}_{\mathrm{g}}\right) \varepsilon_{\mathrm{xc}}\left[\rho\left(\mathbf{r}_{\mathrm{g}}\right)\right] \rho\left(\mathbf{r}_{\mathrm{g}}\right) .
$$

Also, the electron population and charge are partitioned by using the Becke's partitioning function

$$
\begin{gathered}
N_{\mathrm{A}}=\sum_{\mathrm{g}} \omega_{\mathrm{g}} p_{\mathrm{A}}\left(\mathbf{r}_{\mathrm{g}}\right) \rho\left(\mathbf{r}_{\mathrm{g}}\right), \\
q^{\mathrm{A}}=-N_{\mathrm{A}}+Z_{\mathrm{A}} .
\end{gathered}
$$

Blagg-Slater radii are adopted for the Becke's partitioning function. It is noted that the radius used for the hydrogen atom is $0.35 \AA$.

The third group includes the kinetic energy $E_{\mathrm{KIN}}$, local ion core-electron interaction $E_{\mathrm{L}}$, and Coulomb interaction $E_{\mathrm{CLM}}$, which are expressed in the reciprocal space representation. To partition them into atomic contributions, the inverse FFT (IFFT) has been used. The IFFT

$$
f(\mathbf{r})=\frac{1}{\Omega} \sum_{\mathbf{G}} f(\mathbf{G}) \exp (i \mathbf{G} \cdot \mathbf{r}),
$$

allows us to express three energy components $E_{\mathrm{KIN}}, E_{\mathrm{L}}$, and $E_{\mathrm{CLM}}$ in the real-space representation. For example, after the IFFT from reciprocal to real spaces, the kinetic energy is expressed as follows:

$$
E_{\mathrm{KIN}}=\frac{1}{2 \Omega} \sum_{\mathrm{g}} \sum_{i \mathbf{k}} \sum_{\mathbf{G}}\left|c_{\mathbf{k}+\mathbf{G}}^{i}\right|^{2}|\mathbf{k}+\mathbf{G}|^{2} \exp \left(i \mathbf{G} \cdot \mathbf{r}_{\mathrm{g}}\right) .
$$

By inserting the Becke's space-partitioning function, the atomic contribution for atom $\mathrm{A}$ is given as

$$
E_{\mathrm{KIN}}^{\mathrm{A}}=\frac{1}{2 \Omega} \sum_{\mathrm{g}} \omega_{\mathrm{g}} p_{\mathrm{A}}\left(\mathbf{r}_{\mathrm{g}}\right) \sum_{i \mathbf{k}} \sum_{\mathbf{G}}\left|c_{\mathbf{k}+\mathbf{G}}^{i}\right|^{2}|\mathbf{k}+\mathbf{G}|^{2} \exp \left(i \mathbf{G} \cdot \mathbf{r}_{\mathrm{g}}\right)
$$

For Coulomb and ion core-electron interactions, the atomic contributions are given as 


$$
\begin{gathered}
E_{\mathrm{CLM}}^{\mathrm{A}}=2 \pi \Omega \sum_{\mathrm{g}} \omega_{\mathrm{g}} p_{\mathrm{A}}\left(\mathbf{r}_{\mathrm{g}}\right) \sum_{\mathbf{G} \neq 0, \mathbf{G}^{\prime}} \frac{\rho(\mathbf{G})}{|\mathbf{G}|^{2}} \exp \left(-i \mathbf{G} \cdot \mathbf{r}_{\mathrm{g}}\right) \rho\left(\mathbf{G}^{\prime}\right) \exp \left(i \mathbf{G}^{\prime} \cdot \mathbf{r}_{\mathrm{g}}\right), \\
E_{\mathrm{L}}^{\mathrm{A}}=\Omega\left[\frac{1}{2} \sum_{\mathrm{g}} \omega_{\mathrm{g}} p_{\mathrm{A}}\left(\mathbf{r}_{\mathrm{g}}\right) \sum_{\mathbf{G} \neq 0} \rho(\mathbf{G}) \exp \left(i \mathbf{G} \cdot \mathbf{r}_{\mathrm{g}}\right) \sum_{\mathbf{G}^{\prime}} \sum_{\mathrm{B}}^{\text {atom }} V_{\mathrm{B}}^{\mathrm{loc}}\left(\mathbf{G}^{\prime}\right) \exp \left(-i \mathbf{G}^{\prime} \cdot \mathbf{R}_{\mathrm{B}}\right) \exp \left(-i \mathbf{G}^{\prime} \cdot \mathbf{r}_{\mathrm{g}}\right)\right. \\
\left.+\frac{1}{2} \sum_{\mathbf{G} \neq 0} \rho(\mathbf{G}) V_{\mathrm{A}}^{\mathrm{loc}}(\mathbf{G}) \exp \left(-i \mathbf{G} \cdot \mathbf{R}_{\mathrm{A}}\right)\right]
\end{gathered}
$$

Noted that the first and second terms in $E_{\mathrm{L}}^{\mathrm{A}}$ come from contributions from electrons and ion cores. The correction term to the local pseudopotential should be also partitioned in an appropriate way. This energy decomposition scheme is easily applicable to other programs using PW basis functions and pseudopotentials.

\section{NUMERICAL APPLICATIONS}

\section{A. Isolated molecule: $\mathrm{H}_{2} \mathrm{O}$}

First, the PW-EDA method is numerically assessed for an isolated molecule $\mathrm{H}_{2} \mathrm{O}$. We perform three kinds of calculations for comparison. (\#1) All-electron calculations using the Dunning's cc-pVXZ $(X=\mathrm{D}, \mathrm{T}, \mathrm{Q}) \mathrm{GTO}$ basis functions ${ }^{16}$ for $\mathrm{H}$ and $\mathrm{O}$ with the Slater exchange ${ }^{17}$ and Vosko-Wilk-Nusair correlation ${ }^{18}$ (SVWN5) functional. (\#2) Valence-electron calculations using PW basis functions plus the Troullier-Martins norm-conserving pseudopotentials ${ }^{19}$ for $\mathrm{H}$ and $\mathrm{O}$, with the Slater exchange ${ }^{17}$ and Perdew-Wang correlation ${ }^{20}$ (SPW) functional. (\#3) Valence-electron calculations using PW basis functions plus the Troullier-Martins' norm-conserving pseudopotential ${ }^{19}$ for $\mathrm{H}$ and the Vanderbilt's ultrasoft pseudopotential $^{21}$ for $\mathrm{O}$ with the SPW functional.

Geometrical parameters of $\mathrm{H}_{2} \mathrm{O}$ used for \#1-\#3 are optimized at the B3LYP (Refs. 3-5 and 22)/cc-pVQZ (Ref. 16) level of theory. B3LYP means the Becke's three-parameters hybrid functional ${ }^{5,22}$ with the Becke's exchange ${ }^{3}$ and LeeYang-Parr correlation ${ }^{4}$ functionals. In the PW calculations of
$\# 2$ and \#3, $\mathrm{H}_{2} \mathrm{O}$ is located at the center in the unit cell of $20 \times 20 \times 20$ bohr $^{3}$. The cut-off energy $E_{\text {cut }}$ for the wavefunction expansion is changed from 9 to $81 \mathrm{Ry}$, whereas that for the charge-density expansion is set to $400 \mathrm{Ry}$. The $\Gamma$ point is used for Brillouin zone sampling. It should be noted that both SVWN5 and SPW exchange-correlation functionals are categorized into local-density approximation (LDA) but the difference appears in the correlation functional.

The PW-EDA method is applied to the PW calculations of \#2 and \#3. On the other hand, the GTO calculations of \#1 are analyzed by the grid-based EDA (Grid-EDA). ${ }^{14}$ Grid-EDA partitions the total energy into atomic contributions using the Becke's partition function, ${ }^{13}$ which is also adopted for PWEDA. Although the partitioning of Grid-EDA is slightly different from that of PW-EDA owing to the use of the pseudopotentials for PW calculations, the comparison offers useful and interesting information about the difference of the basis sets.

Table I lists atomic-energy contributions estimated by Grid-EDA for the GTO calculations of \#1. $E^{\mathrm{A}}\left(\mathrm{H}_{2} \mathrm{O}\right)$ represents an atomic-energy contribution of atom $\mathrm{A}$ in $\mathrm{H}_{2} \mathrm{O}$ and $\Delta E^{\mathrm{A}}\left(\mathrm{H}_{2} \mathrm{O}\right)$ is a stabilization energy from an atomic state: $E^{\mathrm{A}}\left(\mathrm{H}_{2} \mathrm{O}\right)-E(\mathrm{~A})$, where $E(\mathrm{~A})$ is an atomic energy for atom A. The differences from $E^{\mathrm{A}}\left(\mathrm{H}_{2} \mathrm{O}\right)$ or $\Delta E^{\mathrm{A}}\left(\mathrm{H}_{2} \mathrm{O}\right)$ computed with cc-pVQZ are shown in parentheses in kilocalorie per mole. The cc-pVTZ basis set basically provides the moderately accurate results, which agree with $E^{\mathrm{A}}\left(\mathrm{H}_{2} \mathrm{O}\right)$ (A $=\mathrm{H}, \mathrm{O})$ for the cc-pVQZ basis set within the deviation of 5 $\mathrm{kcal} / \mathrm{mol} . E^{\mathrm{H}}\left(\mathrm{H}_{2} \mathrm{O}\right)$ and $E^{\mathrm{O}}\left(\mathrm{H}_{2} \mathrm{O}\right)$ are estimated to be -0.579

\begin{tabular}{|c|c|c|c|c|}
\hline \multirow[t]{2}{*}{ Entry } & & \multicolumn{3}{|c|}{ Basis set } \\
\hline & & cc-pVDZ & cc-pVTZ & cc-pVQZ \\
\hline \multirow[t]{6}{*}{$\# 1$} & $E^{\mathrm{H}}\left(\mathrm{H}_{2} \mathrm{O}\right)$ & $-0.587(-4.836)$ & $-0.578(0.502)$ & -0.579 \\
\hline & $E^{\mathrm{O}}\left(\mathrm{H}_{2} \mathrm{O}\right)$ & $-74.683(42.170)$ & $-74.742(4.858)$ & -74.750 \\
\hline & $E\left(\mathrm{H}_{2} \mathrm{O}\right)$ & $-75.857(32.499)$ & $-75.899(5.863)$ & -75.908 \\
\hline & $\Delta E^{\mathrm{H}}\left(\mathrm{H}_{2} \mathrm{O}\right)$ & $-68.68(-5.52)$ & $-62.79(0.37)$ & -63.16 \\
\hline & $\Delta E^{\mathrm{O}}\left(\mathrm{H}_{2} \mathrm{O}\right)$ & $-116.30(22.22)$ & $-137.67(0.84)$ & -138.52 \\
\hline & $\Delta E\left(\mathrm{H}_{2} \mathrm{O}\right)$ & $-253.65(11.18)$ & $-263.24(1.59)$ & -264.83 \\
\hline
\end{tabular}

TABLE I. Atomic-energy contributions of $\mathrm{H}_{2} \mathrm{O}$ (in hartree) and its differences from atoms (in $\mathrm{kcal} / \mathrm{mol})$. 


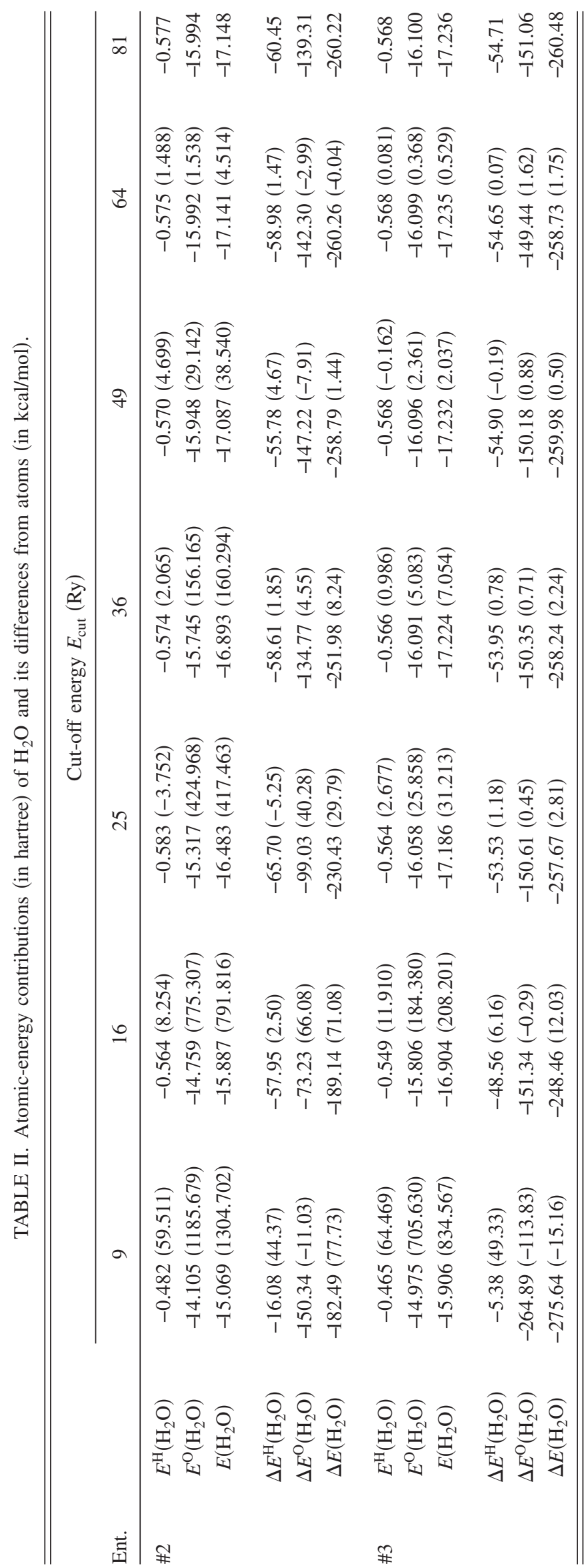

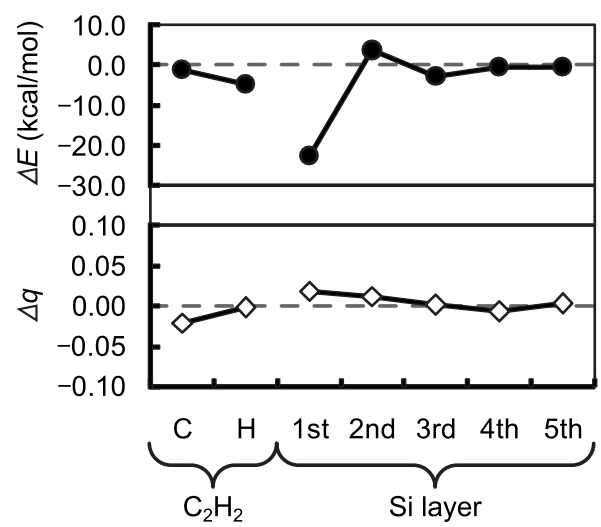

FIG. 1. (a) $\Delta E\left(\mathrm{H}_{2} \mathrm{O}\right)$, (b) $\Delta E^{\mathrm{H}}\left(\mathrm{H}_{2} \mathrm{O}\right)$, and (c) $\Delta E^{\mathrm{O}}\left(\mathrm{H}_{2} \mathrm{O}\right)$ estimated by \#1 with cc-pVXZ $(X=\mathrm{D}, \mathrm{T}, \mathrm{Q})$ and by \#2 and \#3 for $E_{\text {cut }}=9,16,25,36,49,64$, and 81 Ry.

and -74.750 hartree in the case of cc-pVQZ.

Table II lists atomic-energy contributions estimated by PW-EDA for the PW calculations of \#2 and \#3. The differences from $E^{\mathrm{A}}\left(\mathrm{H}_{2} \mathrm{O}\right)$ or $\Delta E^{\mathrm{A}}\left(\mathrm{H}_{2} \mathrm{O}\right)$ computed with $E_{\text {cut }}$ $=81 \mathrm{Ry}$ are shown in parentheses in kilocalorie per mole. For \#2 and \#3, $E^{\mathrm{O}}\left(\mathrm{H}_{2} \mathrm{O}\right)$ slowly converges to that with $E_{\text {cut }}$ $=81 \mathrm{Ry}$, whereas $E^{\mathrm{H}}\left(\mathrm{H}_{2} \mathrm{O}\right)$ less depends on $E_{\text {cut }} \cdot E^{\mathrm{H}}\left(\mathrm{H}_{2} \mathrm{O}\right)$ with $E_{\text {cut }}=81 \mathrm{Ry}$ for \#2 and \#3 are estimated to be -0.577 and -0.568 hartree, which are relatively close to that of \#1, whereas $E^{\mathrm{O}}\left(\mathrm{H}_{2} \mathrm{O}\right)$ is estimated to be -15.994 and -16.100 hartree, which are considerably different from that of \#1 because of the lack of the contributions from core electrons.

Next, we compare the results of $\Delta E\left(\mathrm{H}_{2} \mathrm{O}\right), \Delta E^{\mathrm{H}}\left(\mathrm{H}_{2} \mathrm{O}\right)$, and $\Delta E^{\mathrm{O}}\left(\mathrm{H}_{2} \mathrm{O}\right)$ computed by PW-EDA and Grid-EDA since the energy differences, not the energies, are comparable even for the different type of calculations: all-electron and valence-electron calculations. Figure $1(\mathrm{a})$ shows $\Delta E\left(\mathrm{H}_{2} \mathrm{O}\right)$ estimated in the cases of \#1-\#3. The converged values of $\Delta E\left(\mathrm{H}_{2} \mathrm{O}\right)$ for $\# 1-\# 3$ are $-264.83,-260.22$, and $-260.48 \mathrm{kcal} / \mathrm{mol}$, respectively: \#2 and \#3 provide similar $\Delta E\left(\mathrm{H}_{2} \mathrm{O}\right)$, whereas \#1 yields a slightly larger energy difference in absolute value. This mainly comes from the difference in the correlation functionals used. Let us discuss the convergence. For \#1, $\Delta E\left(\mathrm{H}_{2} \mathrm{O}\right)$ fast and monotonically approaches the converged value with respect to the basis sets. For \#2, $\Delta E\left(\mathrm{H}_{2} \mathrm{O}\right)$ monotonically but slowly approaches the converged value, especially, for $E_{\text {cut }} \geq 49$ Ry. For \#3, $\Delta E\left(\mathrm{H}_{2} \mathrm{O}\right)$ exhibits a fast but damped oscillation. $E_{\text {cut }} \geq 25$ assures the small difference of $3 \mathrm{kcal} / \mathrm{mol}$ with respect to the converged value.

Figure 1(b) illustrates the convergence of $\Delta E^{\mathrm{H}}\left(\mathrm{H}_{2} \mathrm{O}\right)$. The converged values are $-63.16,-60.45$, and $-57.71 \mathrm{kcal} / \mathrm{mol}$ for \#1-\#3, respectively. The GTO basis functions provide the largest stabilization in comparison with $\mathrm{PW}$ basis functions plus pseudopotentials. The difference of $\Delta E^{\mathrm{H}}\left(\mathrm{H}_{2} \mathrm{O}\right)$ is observed in the cases of \#2 and \#3 although $\Delta E\left(\mathrm{H}_{2} \mathrm{O}\right)$ for \#2 and \#3 are estimated to be a similar value, approximately $-260 \mathrm{kcal} / \mathrm{mol}$. For the calculations of \#1 and \#3, $\Delta E^{\mathrm{H}}\left(\mathrm{H}_{2} \mathrm{O}\right)$ monotonically approaches the converged values, whereas it tends to exhibit an oscillating behavior for \#2. The tendency of $\Delta E^{\mathrm{H}}\left(\mathrm{H}_{2} \mathrm{O}\right)$ is different from that of $\Delta E\left(\mathrm{H}_{2} \mathrm{O}\right)$. 

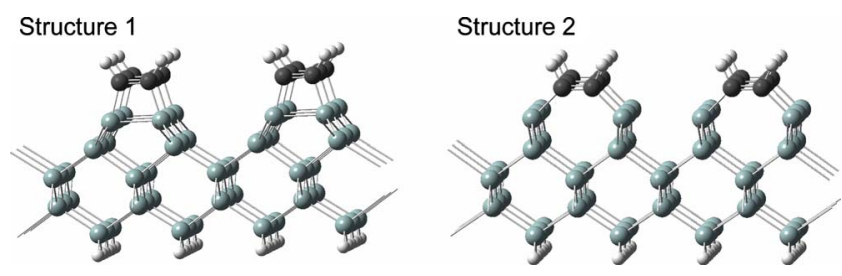

FIG. 2. (Color online) Adsorption structures of $\mathrm{C}_{2} \mathrm{H}_{2}$ on a $\mathrm{Si}(001)-(2 \times 1)$ surface. The optimized geometries are obtained by the PW-PBC calculations with the PBE functional.

Figure 1(c) illustrates the convergence of $\Delta E^{\mathrm{O}}\left(\mathrm{H}_{2} \mathrm{O}\right)$. The converged values are $-138.52,-139.31$, and $-151.06 \mathrm{kcal} / \mathrm{mol}$ for \#1-\#3, respectively. The energy difference in $\Delta E^{\mathrm{O}}\left(\mathrm{H}_{2} \mathrm{O}\right)$ between $\# 2$ and $\# 3$ is $11.75 \mathrm{kcal} / \mathrm{mol}$, which is slightly larger than that for $\Delta E^{\mathrm{H}}\left(\mathrm{H}_{2} \mathrm{O}\right)$. This may be ascribed to the fact that the description of $\mathrm{O}$ atom is different because of the different pseudopotentials. Let us discuss the convergence behavior. A similar tendency is observed: $\Delta E^{\mathrm{O}}\left(\mathrm{H}_{2} \mathrm{O}\right)$ for $\# 1$ and $\# 3$ is numerically stable, whereas it tends to oscillate for \#2.

In general, the partitioned energies, which are not observables, are strongly dependent on the partitioning schemes. In some cases, the partitioned values becomes unphysical and against chemical institution. ${ }^{11,14}$ However, the results compared in Fig. 1 indicate that the present PW-EDA method as well as the previous Grid-EDA offer stable and reasonable partitioned energies.

\section{B. Adsorption system: $\mathrm{C}_{2} \mathrm{H}_{2} / \mathrm{Si}(001)$}

Next, the PW-EDA method is applied to the adsorption system: $\mathrm{C}_{2} \mathrm{H}_{2}$ adsorbed on an $\mathrm{Si}(001)$ surface. We previously examined two models as an adsorption structure: the dimerized model (DM) and dimer-cleaved model (CM) on the $\mathrm{C}_{2} \mathrm{H}_{2} / \mathrm{Si}(001)$ surface, as shown in Fig. 2. This system has attracted much attention in connection with semiconductor materials. Although several adsorption structures and coverage dependence have been extensively examined, ${ }^{23}$ we focus on the two adsorption structures here. A slab $\mathrm{Si}(001)-(2$ $\times 1)$ surface model consisting of five Si layers is adopted, and the $\mathrm{C}_{2} \mathrm{H}_{2}$ molecule adsorbs on the $\mathrm{Si}$ dimer of the first
TABLE III. Bond distances ( $⿱$ A), angles (in degrees), and adsorption energies (in $\mathrm{kcal} / \mathrm{mol}$ ) of the two adsorption structures.

\begin{tabular}{lccccc}
\hline \hline & \multicolumn{2}{c}{ LDA } & & \multicolumn{2}{c}{ GGA } \\
\cline { 2 - 3 } \cline { 5 - 6 } & DM & CM & & DM & CM \\
\hline$R($ Si-Si $)$ & 2.34 & 4.07 & & 2.36 & 4.11 \\
$R(\mathrm{C}-\mathrm{C})$ & 1.35 & 1.36 & & 1.36 & 1.37 \\
$R(\mathrm{Si}-\mathrm{C})$ & 1.89 & 1.91 & & 1.91 & 1.93 \\
$\angle(\mathrm{Si}-\mathrm{C}-\mathrm{C})$ & 105.2 & 135.2 & & 105.2 & 135.2 \\
$\Delta E$ & 55.92 & 80.78 & & 44.61 & 68.62 \\
\hline \hline
\end{tabular}

layer. The edge Si atoms in the fifth layer are terminated with hydrogen atoms. The lattice constant of the Si slabs used in the calculations is set to $3.628 \AA$. All atomic positions except for the fifth layer $\mathrm{Si}$ atoms and the hydrogen atoms for the termination are relaxed. The calculations are carried out with SPW and Perdew-Burke-Ernzerhof (PBE) (Ref. 2) GGA functionals. The norm-conserving ${ }^{19}$ and ultrasof $\mathrm{t}^{21}$ pseudopotentials are adopted for $\{\mathrm{Si}, \mathrm{H}\}$ and $\{\mathrm{C}\}$, respectively. $E_{\text {cut }}$ for the wave-function and charge-density expansions are 25 and $300 \mathrm{Ry}$, respectively. The $32 k$ points in a surface Brillouin zone are used.

As the previous paper has reported, ${ }^{24} \mathrm{DM}$ is energetically preferred to CM by 24.86 and $24.01 \mathrm{kcal} / \mathrm{mol}$ by LDA and GGA where the adsorption energies of $\mathrm{CM}$ and $\mathrm{DM}$ are 55.92 and $80.78 \mathrm{kcal} / \mathrm{mol}$ for LDA and 44.61 and $68.62 \mathrm{kcal} /$ mol for GGA, as shown in Table III. This conclusion has been confirmed by papers. ${ }^{23}$ The $\mathrm{Si}-\mathrm{Si}$ distance for $\mathrm{CM}$ is about $4.10 \AA$, which is significantly longer than about $2.35 \AA$ for DM. The C-C bond distances for both models are approximately $1.36 \AA$, which is close to that of ethylene. This indicates that the $\mathrm{C}-\mathrm{C}$ double bond is formed for both models. The Si-C distances for both models are $\sim 1.9 \AA$. The angle of CCSi for the dimerized structure is $105.2^{\circ}$, which is deviated from $120.0^{\circ}$ of a typical $s p^{2}$ hybrid orbital in spite of the short C-C bond distance. Thus, DM may have a structural strain, which was not clarified in the previous paper. $^{24}$

In order to clarify the difference of the two adsorption structures on the $\mathrm{Si}(001)$ surface, PW-EDA is applied. The following quantities:

TABLE IV. Differences of atomic energies (in $\mathrm{kcal} / \mathrm{mol}$ ) and charges between the two adsorption structures.

\begin{tabular}{|c|c|c|c|c|c|}
\hline & & \multicolumn{2}{|c|}{ LDA } & \multicolumn{2}{|c|}{ GGA } \\
\hline & & $\Delta q^{\mathrm{A}}$ & $\Delta E^{\mathrm{A}}$ & $\Delta q^{\mathrm{A}}$ & $\Delta E^{\mathrm{A}}$ \\
\hline \multirow[t]{2}{*}{$\mathrm{C}_{2} \mathrm{H}_{2}$} & $\mathrm{C}$ & -0.01 & 0.64 & -0.01 & -0.54 \\
\hline & $\mathrm{H}$ & 0.01 & -5.29 & 0.01 & -5.80 \\
\hline \multirow[t]{5}{*}{ Si slab } & First layer & -0.01 & -23.05 & 0.00 & -20.05 \\
\hline & Second layer & -0.01 & 5.22 & -0.01 & 5.03 \\
\hline & Third layer & 0.00 & -1.17 & 0.00 & -1.30 \\
\hline & Fourth layer & 0.01 & -0.93 & 0.01 & -1.07 \\
\hline & Fifth layer & 0.00 & 0.12 & 0.00 & 0.08 \\
\hline Total & & 0 & -24.86 & 0 & -24.01 \\
\hline
\end{tabular}




$$
\Delta q^{\mathrm{A}}=q_{\mathrm{DM}}^{\mathrm{A}}-q_{\mathrm{CM}}^{\mathrm{A}}
$$

and

$$
\Delta E^{\mathrm{A}}=E_{\mathrm{DM}}^{\mathrm{A}}-E_{\mathrm{CM}}^{\mathrm{A}}
$$

are computed. Energy contributions $\Delta E^{\mathrm{A}}$ and charges $\Delta q^{\mathrm{A}}$ of two carbons and two hydrogens in $\mathrm{C}_{2} \mathrm{H}_{2}$, two $\mathrm{Si}$ atoms in each $\mathrm{Si}$ layer are shown in Table IV. Changes in charges of $\mathrm{C}_{2} \mathrm{H}_{2}$ and $\mathrm{Si}$ slab are relatively small: $\left|\Delta q^{\mathrm{A}}\right| \leq 0.02$. It means that the discussion on local stabilities from populations is considerably challenging.

As regards energy contributions, the absolute value of the energy change in the carbon atoms of $\mathrm{C}_{2} \mathrm{H}_{2}$ is less than 1.0 $\mathrm{kcal} / \mathrm{mol}$, while that of the hydrogen atoms is close to $-5.0 \mathrm{kcal} / \mathrm{mol}$. The sum of the stabilization energy of the $\mathrm{C}_{2} \mathrm{H}_{2}$ part is -5.93 and $-6.34 \mathrm{kcal} / \mathrm{mol}$ for LDA and GGA, respectively, which means that structural strain does not affect the stability of the $\mathrm{C}_{2} \mathrm{H}_{2}$ part. Let us examine the Si slab part. Energy changes are large for the Si first layer: -23.05 and $-20.05 \mathrm{kcal} / \mathrm{mol}$ for LDA and GGA, respectively. The stabilization from the first $\mathrm{Si}$ layer is a dominant factor. $\Delta E^{\mathrm{Si}}$ of the second $\mathrm{Si}$ layer is estimated to be approximately 5 $\mathrm{kcal} / \mathrm{mol}$, which indicates that the stability in the second $\mathrm{Si}$ layer of $\mathrm{CM}$ is larger than that of DM. The third layer reverses the trend: $\Delta E^{\mathrm{Si}}$ is -1.17 and $-1.30 \mathrm{kcal} / \mathrm{mol}$ for LDA and GGA, respectively. $\left|\Delta E^{\mathrm{Si}}\right|$ of the fourth and fifth layers are at most $1.0 \mathrm{kcal} / \mathrm{mol}$. The sum of the stabilization energy of the Si slab part is -19.82 and $-17.31 \mathrm{kcal} / \mathrm{mol}$ for LDA and GGA, respectively. Thus, the Si slab plays a dominant role in determining the stable structure. The above analysis demonstrates that the main factor of the stabilization of DM is ascribed to the stabilization of the first Si layer, not the structural strain of the carbon atoms in $\mathrm{C}_{2} \mathrm{H}_{2}$. This application indicates that the adsorption can be understood in terms of local energy changes estimated by PW-EDA even though charge variations are relatively small.

\section{CONCLUDING REMARKS}

We formulate and implement an analysis method, called PW-EDA that partitions into atomic contributions the total energy calculated with PW basis functions and pseudopotentials. PW-EDA is applied to isolated and adsorption systems: $\mathrm{H}_{2} \mathrm{O}$ and $\mathrm{C}_{2} \mathrm{H}_{2} / \mathrm{Si}(001)$. The PW-EDA results of $\mathrm{H}_{2} \mathrm{O}$ demonstrate the cut-off energy dependence of atomic-energy contributions. We also reveal that PW-EDA and Grid-EDA provide similar atomic contributions in spite of the difference in the energy-partitioning schemes. The PW-EDA results of the adsorption of $\mathrm{C}_{2} \mathrm{H}_{2}$ on the $\mathrm{Si}(001)$ surface clarify why the dimerized structure is more stable than the dimer-cleaved structure. The numerical applications confirm the reliability and usefulness of the PW-EDA method. PW-EDA offers a powerful tool to examine local effects in extended systems and provides interpretations on phenomena in terms of energy stability.

\section{ACKNOWLEDGMENTS}

We are grateful to Takenori Yamamoto for a fruitful discussion. Part of the calculations was performed at the Research Center for Computational Science (RCCS), Okazaki Research Facilities, National Institutes of Natural Sciences (NINS). This study was partially supported by a Grant-inAid for Scientific Research on Priority Areas "Molecular Theory for Real Systems" "KAKENHI (Grant No. 18066016)" from the Japanese Ministry of Education, Culture, Sports, Science and Technology (MEXT), Japan, by a Grant-in-Aid for Young Scientists (Start-up) "KAKENHI (Grant No. 19850027)" from Japanese Society for the Promotion of Science (JSPS), by Global Center of Excellence (GCOE) "Practical Chemical Wisdom" from the MEXT, by Nanoscience Program in the Next Generation Super Computing Project of the MEXT, and by the project research grant "Development of high-performance computational environment for quantum chemical calculation and its assessment" from the Advanced Research Institute for Science and Engineering (RISE), Waseda University. This paper is part of the outcome of research performed under Waseda University Grants for Special Research Projects (Grant Nos. 2009B-102 and 2009B-163).

\footnotetext{
*Corresponding author; nakai@waseda.jp

${ }^{1}$ R. Car and M. Parrinello, Phys. Rev. Lett. 55, 2471 (1985).

${ }^{2}$ J. P. Perdew, K. Burke, and M. Ernzerhof, Phys. Rev. Lett. 77, 3865 (1996); 78, 1396 (1997).

${ }^{3}$ A. D. Becke, Phys. Rev. A 38, 3098 (1988).

${ }^{4}$ C. Lee, W. Yang, and R. G. Parr, Phys. Rev. B 37, 785 (1988).

${ }^{5}$ A. D. Becke, J. Chem. Phys. 98, 5648 (1993).

${ }^{6}$ C. Pisani and R. Dovesi, Int. J. Quantum Chem. 17, 501 (1980).

${ }^{7}$ V. R. Saunders, Faraday Symp. Chem. Soc. 19, 79 (1984).

${ }^{8}$ R. Dovesi, B. Civalleri, R. Orlando, C. Roetti, and V. R. Saunders, in Reviews in Computational Chemistry, edited by K. B. Lipkowitz, R. Larter, and T. R. Cundari (Wiley, New York, 2005), Vol. 21, Chap. 1.

${ }^{9}$ K. N. Kudin and G. E. Scuseria, Phys. Rev. B 61, 16440 (2000).
}

${ }^{10}$ R. S. Mulliken, J. Chem. Phys. 23, 1833 (1955).

${ }^{11}$ H. Nakai, Chem. Phys. Lett. 363, 73 (2002); Y. Kawamura and H. Nakai, J. Comput. Chem. 25, 1882 (2004); H. Nakai, Y. Kurabayashi, M. Katouda, and T. Atsumi, Chem. Phys. Lett. 438, 132 (2007); Y. Kikuchi, Y. Imamura, and H. Nakai, Int. J. Quantum Chem. 109, 2464 (2009); T. Baba, M. Takeuchi, and H. Nakai, Chem. Phys. Lett. 424, 193 (2006).

${ }^{12}$ H. Nakai and Y. Kikuchi, J. Theor. Comput. Chem. 4, 317 (2005); Y. Imamura, T. Baba, and H. Nakai, Int. J. Quantum Chem. 108, 1316 (2008).

${ }^{13}$ A. D. Becke, J. Chem. Phys. 88, 2547 (1988).

${ }^{14}$ Y. Imamura, A. Takahashi, and H. Nakai, J. Chem. Phys. 126, 034103 (2007).

${ }^{15}$ The PHASE code is developed within the RSS21 project sup- 
ported by the Japanese Ministry of Education, Culture, Sports, Science and Technology (MEXT), Japan. See the website http:// www.ciss.iis.u-tokyo.ac.jp/rss21/en/

${ }^{16}$ T. H. Dunning, Jr., J. Chem. Phys. 90, 1007 (1989).

${ }^{17}$ J. C. Slater, Phys. Rev. 81, 385 (1951).

${ }^{18}$ S. H. Vosko, L. Wilk, and M. Nusair, Can. J. Phys. 58, 1200 (1980).

${ }^{19}$ N. Troullier and J. L. Martins, Phys. Rev. B 43, 1993 (1991).

${ }^{20}$ J. P. Perdew and Y. Wang, Phys. Rev. B 45, 13244 (1992).
${ }^{21}$ D. Vanderbilt, Phys. Rev. B 41, 7892 (1990); K. Laasonen, A. Pasquarello, R. Car, C. Lee, and D. Vanderbilt, ibid. 47, 10142 (1993).

${ }^{22}$ P. J. Stephens, F. J. Devlin, C. F. Chabalowski, and M. J. Frisch, J. Phys. Chem. 98, 11623 (1994).

${ }^{23}$ Y. Morikawa, Phys. Rev. B 63, 033405 (2001); D. C. Sorescu and K. D. Jordan, J. Phys. Chem. B 104, 8259 (2000).

${ }^{24}$ Y. Imamura, Y. Morikawa, T. Yamasaki, and H. Nakatsuji, Surf. Sci. 341, L1091 (1995). 